\title{
Développement de solutions hyperfréquences pour la société moderne : Bio-radar et Microscopie électromagnétique
}

\author{
K. Haddadi, D. Szymik, V. Hoel et G. Dambrine \\ Université de Lille, CNRS, UMR 8520 - IEMN F-59000 Lille, France \\ Pôle CNFM de Lille \\ Contact email : kamel.haddadi@univ-lille.fr
}

Cet article décrit le projet de fin d'étude mis au point au sein du Master Réseaux et Télécommunications parcours SYSCOM (Systèmes Communicants) de l'Université de Lille. Le projet vise à développer des solutions hyperfréquences pour la ville intelligente (Smart City). Les développements techniques et scientifiques reposent sur la plate-forme hyperfréquence du GIP-CNFM de Lille qui comprend toutes les installations nécessaires pour mener à bien un projet complet comprenant l'analyse, la conception et la réalisation de composants et circuits hyperfréquences. En particulier, deux projets menés de concert sont décrits. Le premier projet vise à développer un radar hyperfréquence monostatique opérant à $6,2 \mathrm{GHz}$ pour la mesure sans contact des fréquences respiratoire et cardiaque d'un individu. Le deuxième projet ambitionne de développer un microscope électromagnétique original pour le Contrôle Non Destructive (CND) de matériaux à haute résolution spatiale. Un cahier des charges en terme de coût, de performances de mesure et de possibilités d'intégration est défini préalablement avec pour objectif de développer des solutions économiques viables.

\section{Introduction}

L'une des principales applications de l'électromagnétisme est la conception et la réalisation de circuits et de systèmes électroniques dans la gamme des fréquences microondes $(300 \mathrm{MHz}-300 \mathrm{GHz})$ (1). La technologie hyperfréquence a traversé des périodes de changements révolutionnaires, incluant les structures coaxiales et guides d'ondes, les circuits hybrides et monolithiques (2). Ces évolutions technologiques ont modifié les outils requis par les ingénieurs pour la conception et la fabrication de circuits hyperfréquences. Par conséquent, l'enseignement de l'ingénierie par micro-ondes s'avère fondamental pour les étudiants dans les spécialités de la radio et de la communication (3)-(4).

Dans cet article nous décrivons un système de formation pour l'ingénierie micro-onde basé sur l'utilisation de la plate-forme hyperfréquence du CNFM de la Faculté des Sciences et Technologies de Lille (GIP-CNFM Lille) (5). Le GIP-CNFM Lille est largement reconnu dans le domaine des applications radiofréquences et hyperfréquences. Les savoir-faire bâtis autour de compétences et d'expertises pluridisciplinaires lui permettent d'offrir une offre de formation complète en travaux de conception, mise au point et production de composants aux sous-systèmes électroniques. Cette expertise du pôle lillois bénéficie de plates-formes de conception assistée par ordinateur (CAO), d'une salle blanche et d'équipements de test et de contrôle réparties sur une surface de $3.000 \mathrm{~m}^{2}$.

This is an Open Access article distributed under the terms of the Creative Commons Attribution License (http://creativecommons.org/licenses/by/4.0), which permits unrestricted use, distribution, and reproduction in any medium, provided the original work is properly cited. 
Des projets innovants définis dans le cadre de la ville intelligente (Smart City) sont proposés aux étudiants du Master Réseaux et Télécommunications. Rappelons que ce terme de ville intelligente est un concept de développement urbain. Il s'agit d'améliorer la qualité de vie des citadins en rendant la ville plus adaptative et efficace, à l'aide de nouvelles technologies qui s'appuient sur un écosystème d'objets et de services. Le premier projet vise à développer un radar de mesure des fréquences respiratoires et cardiaques de l'individu. Ce système répond à des besoins réels et variés identifiés par la société moderne décrits dans la suite de cet article. Le deuxième projet vise à développer un microscope champ-proche hyperfréquence pour l'imagerie à haute résolution spatiale. Ainsi, ce deuxième projet s'inscrit dans le thème général du CND qui consiste à examiner un objet ou un matériau de telle manière qu'à l'issue de ce contrôle, son utilisation future n'en soit pas affectée. A ce titre, le marché mondial du CND représente15 milliards $€$ en 2016 et devrait représenter 24 milliards $€$ en 2023 (6).

Le contexte et l'organisation des projets sont décrits en section II. Les projets sont développés respectivement en sections III et IV.

\section{Contexte et organisation}

Les deux projets nécessitent une durée qui s'étale sur les deux semestres. Ainsi, les enseignements traditionnels dispensés au sein du cursus universitaire apportent les compléments théoriques et pratiques complémentaires. En particulier, les étudiants se familiarisent avec les techniques radar et les circuits micro-ondes. Les circuits hyperfréquences actifs abordés dans le cours comprennent les amplificateurs, les oscillateurs, les mélangeurs et les détecteurs de puissance.

Les étudiants sont encadrés par une équipe pédagogique composée de trois enseignants chercheurs et d'un ingénieur 'Support technique enseignement Electronique'. Une formation en communication est dispensée préalablement pour optimiser la communication étudiants - enseignants. Cette dernière repose principalement sur la tenue de rapports techniques et d'avancement, des présentations ppt. pour la discussion technique et pour la communication extérieure.

Un accompagnement soutenu dans les premières phases du projet est mis en place pour la présentation du projet et la formation sur les outils de conception. L'ingénieur 'Support technique' est le relais indispensable entre les étudiants et le corps enseignant chercheur. En particulier, la commande de matériels et de composants, la formation sur les outils de réalisation en salle blanche et les équipements de tests et de mesures sont assurés par un personnel disponible, formé et dédié. Ainsi, les étudiants amenés à travailler en autonomie sont confrontés aux différentes facettes des métiers de l'ingénieur hyperfréquence.

\section{III.Développement d'un bio-radar}

\section{Contexte Smart City}

Le projet est défini en relation étroite avec le monde médical et implique un médecin (Dr N. Ghelboun, Faculté de Médecine Henri Warembourg, Université de Lille) en tant que partenaire externe. Par conséquent, la première étape du projet consiste à définir les spécifications du système par le biais d'une réunion entre les étudiants, le corps enseignant et le médecin. Les principales conclusions sont données ci-après. La mesure de la fréquence respiratoire est toujours intéressante pour détecter les maladies respiratoires, surtout sans contact, car elle permet de gagner du temps pour le médecin. La détection du stress est principalement liée à la fréquence cardiaque, la respiration s'accélérant après un certain temps. Le capteur s'avère être une solution innovante pour surveiller les nourrissons et 
éviter la mort subite en milieu hospitalier. Le système peut trouver des applications pour réveiller les personnes après un sommeil paradoxal où la respiration est plus rapide. Le système est intéressant dans les services gériatriques ou des grands brulés où il est difficile de manipuler les patients. Cet échange préliminaire démontre également l'intérêt de la solution proposée. En effet, la plupart des techniques de mesure existantes nécessitent le port d'un appareil (cardiofréquencemètre, oxymètre).

\section{Cahier des charges}

Un cahier des charges en termes de coût réduit et de compacité est défini dans l'objectif d'offrir une solution viable économiquement. Ainsi, nous optons pour une réalisation en technologie plaquée. Le dispositif opère à la fréquence de $6,2 \mathrm{GHz}$ en dehors des fréquences de télécommunications susceptibles de perturber le dispositif.

Le système radar se compose de cinq circuits hyperfréquences, i.e. un oscillateur commandé en tension, un coupleur de type 'BranchLine', une antenne, un amplificateur faible bruit et un détecteur de puissance (figure 1). Le système est complété par un bloc de conditionnement et d'analyse numérique du signal. L'oscillateur génère un signal à onde continue. Le système fonctionne en mode monostatique : une seule antenne transmet et reçoit les signaux hyperfréquences. L'architecture proposée s'affranchit de l'utilisation de commutateur ou de circulateur volumineux et coûteux que l'on trouve couramment dans les configurations de radar (7). Le coupleur sépare l'onde émettrice de celle reçue. Ainsi, le coupleur délivre le signal transmis à l'antenne et injecte le signal diffusé par la cible au détecteur de puissance après amplification. Le signal résultant est donc directement converti en tension continue. La tension continue directement proportionnelle au mouvement de la cible (respiratoire et cardiaque) est ensuite numérisée et traitée par transformée de Fourier rapide (FFT).

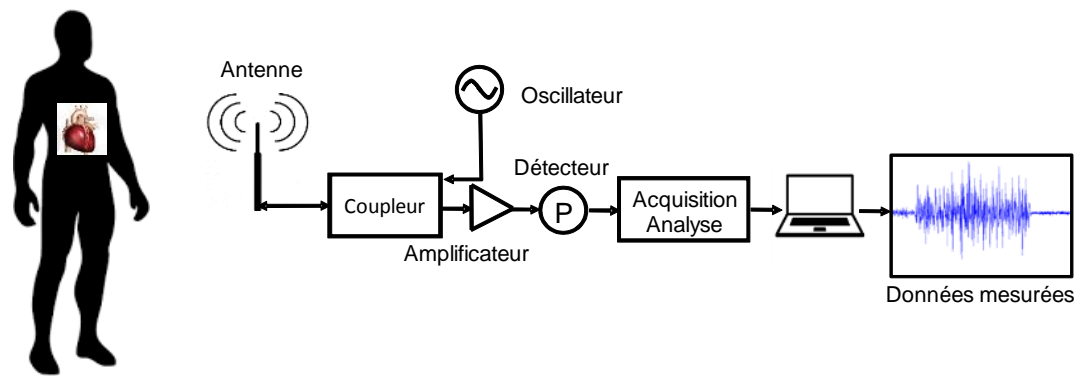

Fig.1. Synoptique du bio-radar opérant à $6,2 \mathrm{GHz}$ pour la mesure simultanée des fréquences respiratoires et cardiaques de l'individu.

Nous décrivons dans la suite les étapes de conception, de réalisation et de tests des circuits coupleur, antennes et amplificateur faible bruit. Au préalable, une étape de calibrage du substrat hyperfréquence est entreprise. Cette étape permet de déterminer la permittivité complexe des substrats afin d'alimenter la plate-forme de CAO KeysightADS ${ }^{\circ}$. Cette première étape permet, par ailleurs, d'évaluer expérimentalement les limitations inhérentes au procédé de fabrication technologique par lithographie optique.

Etape 1 - Calibrage des substrats : Si les plates-formes de CAO offrent des utilitaires pour le dimensionnement des structures de propagation, l'étude est réalisée préalablement au travers de l'utilisation des équations d'Hammerstad afin de familiariser l'étudiant avec le formalisme mathématique implémenté dans les outils de CAO. Nous optons pour un substrat Téflon dont la permittivité relative constructeur est spécifiée à 2,55 et dont le coût 
est de $30 €$ par $\mathrm{dm}^{2}$ pour le projet bio-radar. De la même manière, nous optons pour un substrat en verre Epoxy dont la permittivité relative est donnée à 4,5 et dont le coût est inférieur à $6 €$ par $\mathrm{dm}^{2}$ pour le projet microscopie. A titre d'illustration, nous donnons les étapes et résultats du calibrage du substrat concernant le verre Epoxy en figure 2.

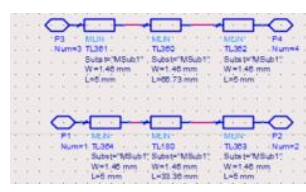

(a)

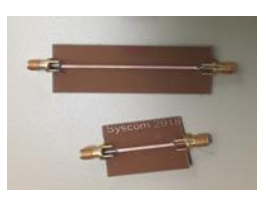

(b)

\begin{tabular}{|c|c|}
\hline Épaisseur du substrat & $\mathrm{H}=800 \mathrm{um}$ \\
\hline Constante diélectrique relative & $\mathrm{Er}=4,19$ \\
\hline Constante de permittivité relative & $\mathrm{Mur}=1$ \\
\hline Conductivité & $\mathrm{Cond}=10^{35}$ \\
\hline Épaisseur du conducteur & $\mathrm{T}=35 \mathrm{um}$ \\
\hline Tangente Delta (pertes diélectriques) & $\mathrm{TanD}=0,023$ \\
\hline
\end{tabular}

(c)

Fig.2. Calibrage du substrat hyperfréquence en verre Epoxy. (a) Dimensionnement des structures de propagation sous ADS. (b) Fabrication des structures de propagation (2 lignes de longueurs différentes sont considérées pour s'affranchir des effets des connecteurs coaxiaux. (c) Calibrage des propriétés diélectriques à $2,45 \mathrm{GHz}$ obtenue par rétro-simulation des données mesurées à l'analyseur de réseaux vectoriel.

Etape 2 - Coupleur hybride $-3 \mathrm{~dB} /-90^{\circ}$ de type 'BranchLine' : le dispositif est constitué de 4 branches de longueurs $\lambda_{\mathrm{g}} / 4$ (où $\lambda_{\mathrm{g}}$ représente la longueur d'onde guidée) comme illustré en figure 3(a). Les impédances caractéristiques des tronçons quart d'onde sont choisies de manière à obtenir une équipartition de la puissance injectée (en voie 1) sur les voies directes (2) et (3). La quatrième voie dénommée « isolée » présente un coefficient de transmission $S_{41}$ théoriquement nul. Le circuit réalisé est donné en figure 3(b). Des transitions coaxiales/micro-ruban soudées sur les ports du dispositif permettent sa caractérisation par l'utilisation d'un analyseur de réseaux vectoriel.
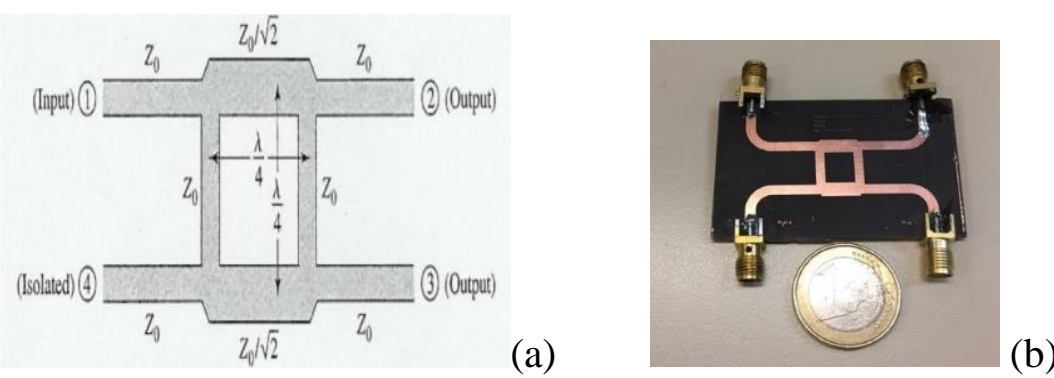

Fig.3. Coupleur Branchline $-3 \mathrm{~dB} /-90^{\circ}$. (a) Synoptique du coupleur. (b) Coupleur réalisé en technologie micro-ruban à 6,2 GHz.

Nous présentons, à titre d'illustration, les résultats de simulation et de mesure relatifs aux déphasages obtenus par la simulation et par la mesure dans la bande de fréquences 4 - $8 \mathrm{GHz}$. Nous notons un déphasage d'environ $90^{\circ}$ entre les voies directes et couplées.

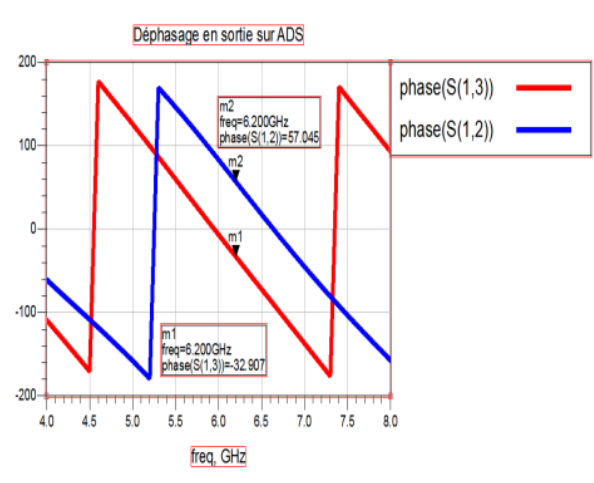

(a)

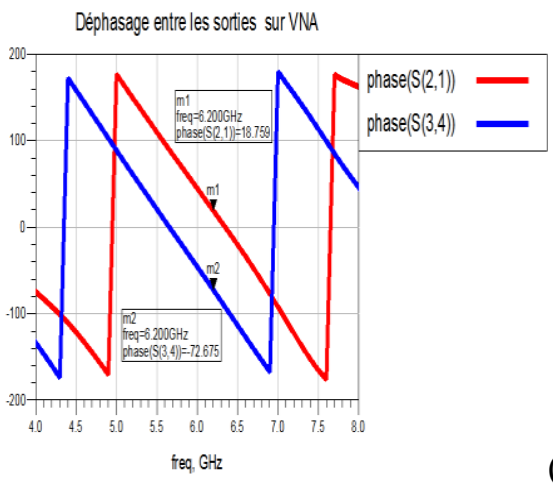

(b)

Fig.4. Comparaison des déphasages en sortie du coupleur obtenus par (a) simulation ADS et (b) mesures à l'analyseur de réseaux vectoriels. 
Etape 3 - Antenne : un travail préalable de conception d'antennes élémentaires de type patch est entrepris au travers des simulateurs électromagnétiques Momentum ${ }^{\circledR}$ 3D et CST Microwave Studio ${ }^{\circledR}$. A l'issue des étapes de fabrication et de tests (adaptation, diagramme de rayonnement), un réseau d'antennes est conçu, réalisé et validé expérimentalement avec des performances de $-35 \mathrm{~dB}$ en adaptation, un angle d'ouverture de $13^{\circ}$ à $-3 \mathrm{~dB}$ et un gain de $12 \mathrm{~dB}$ à $6,2 \mathrm{GHz}$ (figure 5).

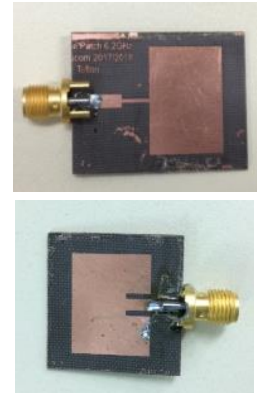

(a)

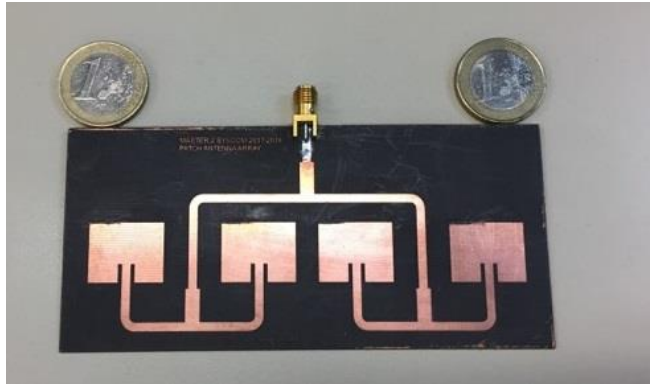

(b)

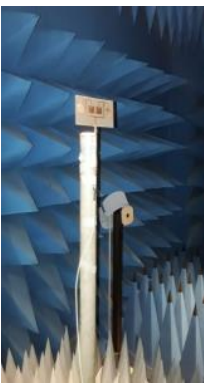

(c)

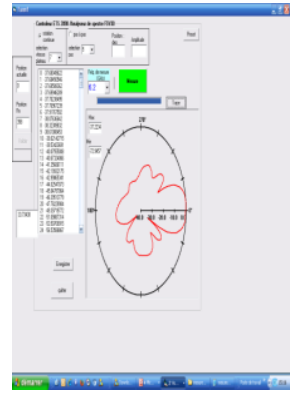

(d)

Fig.5. Antennes à 6,2 GHz (a) Antennes élémentaires. (b) Réseau de 4 antennes patchs. (c) Configuration de mesure en chambre anéchoïque. (d) Diagramme de rayonnement expérimental.

Etape 4 - La conception de l'amplificateur faible bruit dans l'environnement ADS est basée sur le transistor MGF1302 (AsGa). Le cahier des charges inclut un minimim de facteur de bruit fixé à 2 , un gain de $10 \mathrm{~dB}$ ainsi qu'une adaptation en sortie de $-15 \mathrm{~dB}$. Les étapes de conception regroupent la polarisation du transistor, la stabilisation par contreréaction entrée-sortie et des circuits d'adaptation quart d'onde (figure 6).

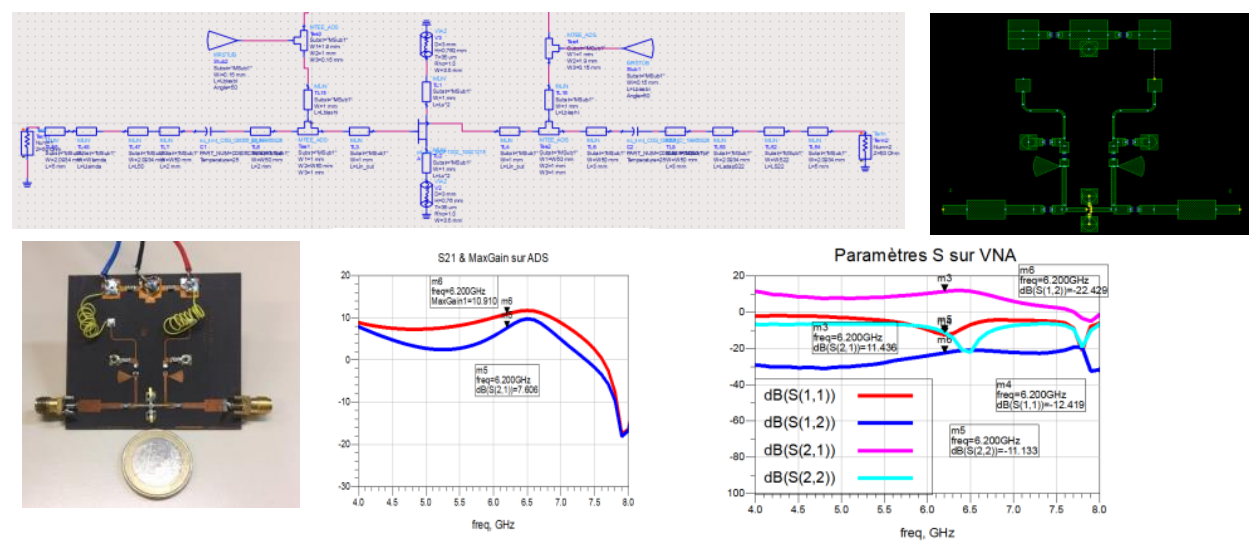

Fig.6. Amplificateur faible bruit à $6,2 \mathrm{GHz}$ (a) Schéma de conception ADS. (b) Masque du circuit. (c) Photographie de l'amplificateur. (d) Simulation ADS en fonction de la fréquence. (e) Mesures des paramètres $S$ en fonction de la fréquence.

Etape 5 - Intégration système : le système complet utilise un module d'acquisition de type Analog Discovery ${ }^{\circledR}$ pour la conversion analogique numérique. Le traitement numérique (FFT) est réalisé dans l'environnement MATLAB®. La figure 7 illustre le banc de test ainsi qu'un exemple de mesure de signes vitaux effectuée à une distance antenneindividu de $10 \mathrm{~cm}$. 


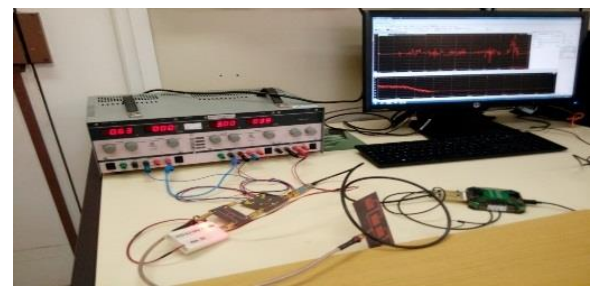

(a)

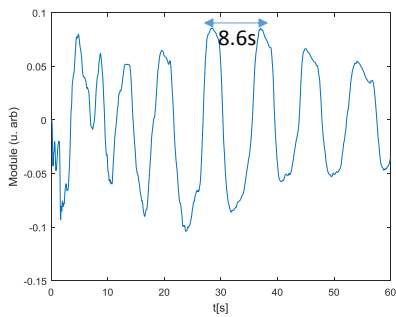

(b)

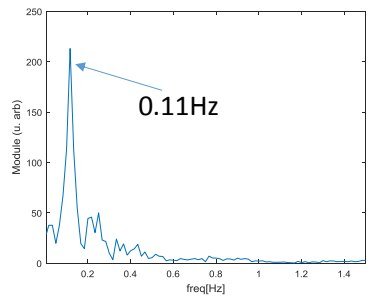

(c)

Fig.7. Bio-radar à 6,2 GHz (a) Photographie du banc de test. (b) Mesure brute du signal détecté en fonction du temps (durée $=60$ secondes). (c) Transformée de Fourier rapide (FFT) du signal détecté.

Afin d'éprouver le système, une campagne de mesures considérant un obstacle (planche de bois d'épaisseur $5 \mathrm{~cm}$ ) est positionné entre le bio-radar et la cible. Nous relevons la réponse temporelle en considérant un temps d'acquisition d'environ 10 secondes. Deux distances antenne / cible fixées à $25 \mathrm{~cm}$ (Fig. 8) et $45 \mathrm{~cm}$ (Fig. 9) sont considérées.

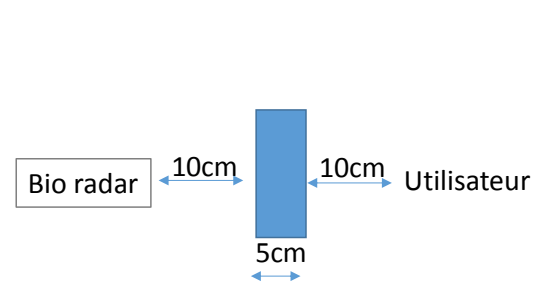

(a)

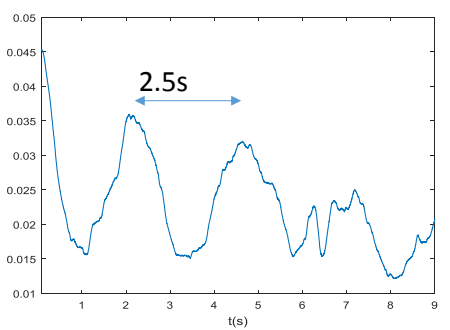

(b)

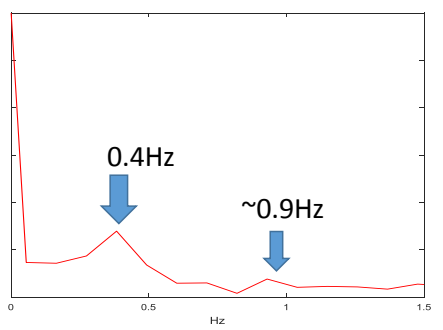

(c)

Fig.8. (a) Configuration de mesure avec obstacle en considérant une distance radar / cible de $25 \mathrm{~cm}$. (b) Réponse temporelle (unités arbitraires). (c) Transformée de Fourier Rapide (FFT) du signal temporel.

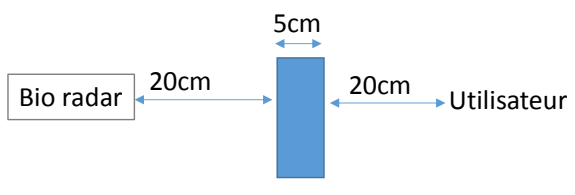

(a)

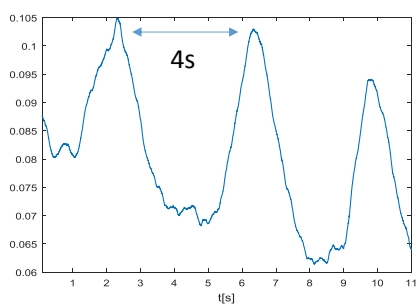

(b)

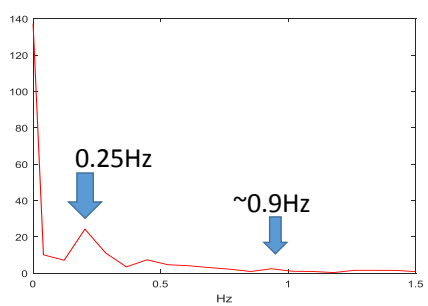

(c)

Fig.9. (a) Configuration de mesure avec obstacle en considérant une distance radar / cible de $45 \mathrm{~cm}$. (b) Réponse temporelle (unités arbitraires). (c) Transformée de Fourier rapide (FFT) du signal temporel.

Pour les deux cas considérés, nous notons une bonne sensibilité de mesure. La transformée de Fourier rapide (FFT) permet d'extraire les pics de fréquence liés à l'activité respiratoire, respectivement à $0,4 \mathrm{~Hz}$ et $0,25 \mathrm{~Hz}$. Nous attribuons également l'existence d'un deuxième pic fréquentiel de niveau très faible à l'activité cardiaque, à $0,9 \mathrm{~Hz}$.

\section{Développement d'un microscope électromagnétique}

\section{Contexte Smart City}

Nous abordons ici le développement du microscope électromagnétique opérant à la fréquence ISM (Industriel, Scientifique et Médical) de 2,45 GHz. C'est dans ce contexte que le système proposé se démarque des techniques conventionnelles de contrôle nondestructif (CND) (ultrasons, radiographie, courants de Foucault, optique) en offrant une mesure sans contact, non invasive, non ionisante, à très faible puissance et à très haut pouvoir de résolution spatiale. En effet, un microscope électromagnétique est généralement 
constitué d'une sonde de dimension très inférieure à la longueur d'onde de propagation qui est reliée à un analyseur de réseaux vectoriel pour la mesure du coefficient de réflexion. Cette pointe est placée à proximité de l'échantillon afin que l'interaction entre la sonde et l'échantillon soit maximale. Dans ce cas, les champs électromagnétiques interagissent avec l'échantillon, ce qui induit une variation de l'impédance de la sonde champ proche. Cette variation est mesurée par l'analyseur de réseaux.

\section{Cahier des charges}

Le cahier des charges repose sur la fabrication d'un microscope s'affranchissant de l'utilisation de l'analyseur de réseaux afin d'offrir des solutions de CND compactes et à faible coût. L'objectif principal est donc la conception d'un réflectomètre pour la mesure de coefficients de réflexion complexes à $2,45 \mathrm{GHz}$. Le dispositif est complété par une sonde de mesure microruban effilée à une dimension sub-longueur d'onde pour réaliser l'imagerie de défauts sub-surfaciques de matériaux. Nous optons pour la conception d'un réflectomètre utilisant la technologie six-ports (8). L'architecture du circuit utilise uniquement un réseau passif à six accès constitué de diviseurs de puissance Wilkinson et de coupleurs 'Branchline' (figure 10). La détection de puissance est réalisée par quatre détecteurs de puissance reposant sur l'utilisation de diodes Schottky 'zero bias' opérant en régime quadratique (non représentés dans ce manuscrit). Le travail a consisté à concevoir, optimiser et réaliser les éléments constitutifs du réflectomètre ainsi que la sonde microruban. Après validation, l'intégration du système est réalisée afin de fournir une solution compacte (figure 10). Le système complet inclut un scanner XYZ pour le déplacement du matériau à imager sous la sonde de mesure.

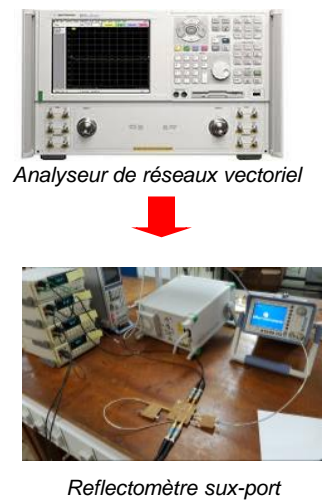

(a)

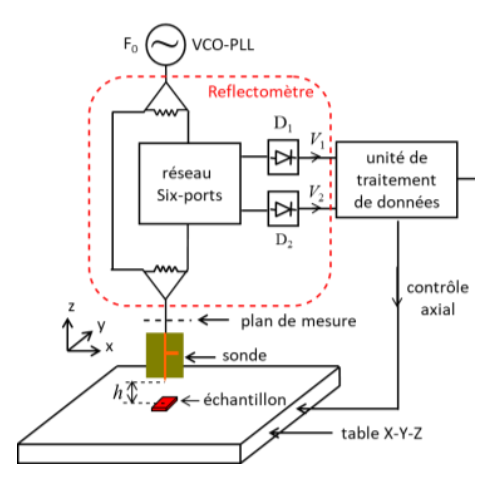

(b)

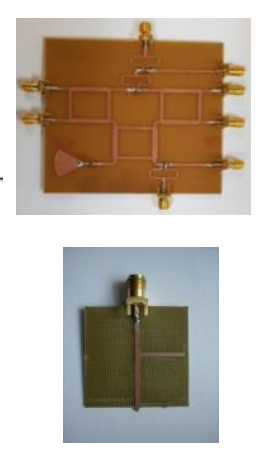

(c)

Fig.10. Architecture du microscope électromagnétique. (a) Comparaison entre analyseur de réseaux vectoriel et réflectomètre six-ports. (b) Synoptique du microscope électromagnétique. (c) Photographies du réflectomètre six-ports et de la sonde micro-ruban sub-longueur d'onde.

Dans la suite de ce manuscrit, nous nous focalisons sur le calibrage vectoriel du dispositif six-ports qui requiert des considérations particulières. En effet, la mesure calibrée du coefficient de réflexion complexe du dispositif sous test nécessite la formulation du problème direct, i.e. expression mathématique des tensions détectées en fonction des parties réelle et imaginaire du coefficient de réflexion. Le lecteur est invité à consulter les références (9)-(15) pour les développements mathématiques relatifs à l'établissement du modèle direct. Le banc de mesure expérimental, décrit en figure 11, vise à relever les tensions détectées en fonction de charges étalons (court-circuit variable et charge adaptée) préalablement mesurées à l'analyseur de réseaux vectoriel. 


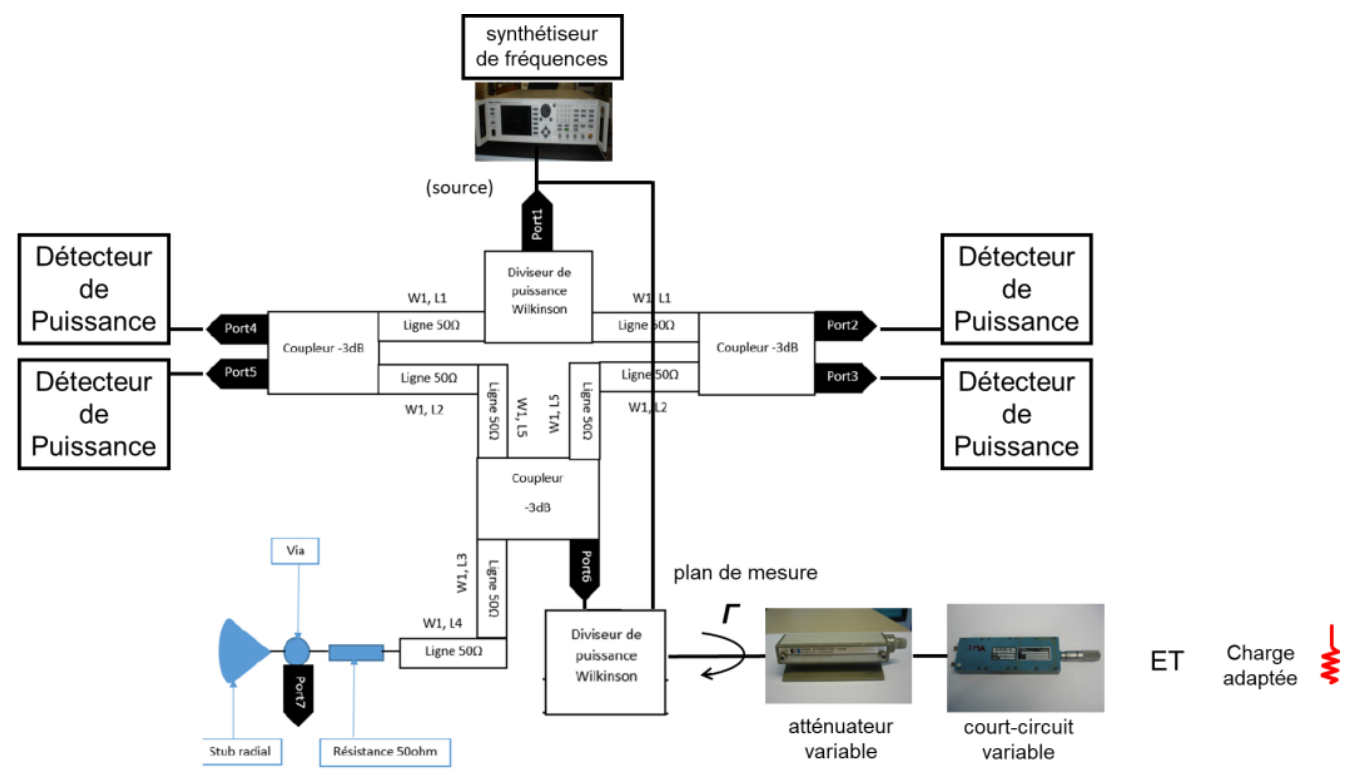

Fig.11. Banc de mesure expérimental pour l'étalonnage du réflectomètre six-ports.

Les figures 12(a) et 12(b) représentent les tensions détectées respectivement sur les ports 2 et 4 (Figure 11). L'onde réfléchie par le dispositif sous test de coefficient de réflexion $\Gamma$ présente un retard de phase de $-90^{\circ}$ entre les trajets Port 6 / Port 2 et Port 6 / Port 4. Incidemment, les réponses mesurées par les détecteurs en fonction de la position du courtcircuit variable (déphasage total de $360^{\circ}$ ) sont en quadrature. Par conséquent, les réponses mesurées, signatures des composantes réelle I et imaginaire Q du coefficient de réflexion complexe, permettent de déterminer $\Gamma$.

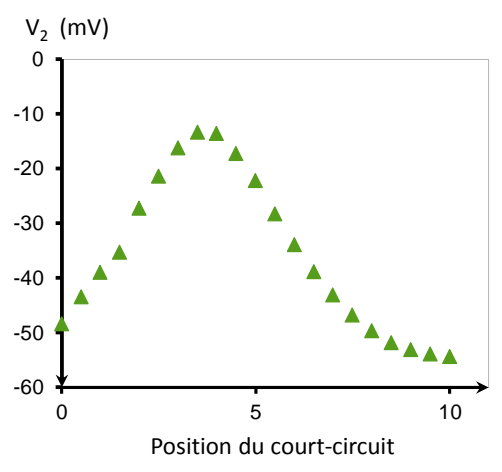

(a)

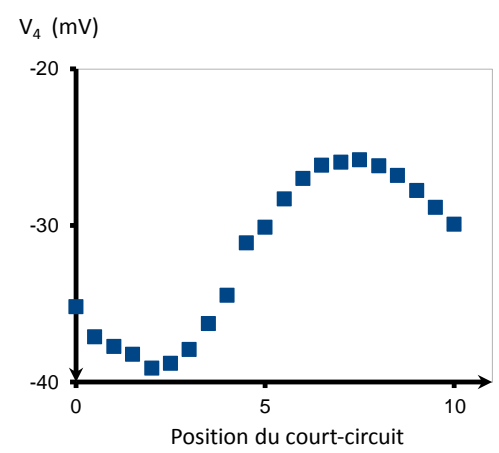

(b)

Fig.12. Tensions détectées en fonction de la position du court-circuit variable.

\section{Conclusion}

Deux projets hyperfréquences développés dans le cadre du Master Réseaux et Télécommunications parcours SYSCOM (Systèmes Communicants) de l'Université de Lille sont décrits dans cet article. Ces projets s'inscrivent dans le développement de solutions matérielles et logicielles pour la ville intelligente. Réalisés au sein de la plateforme hyperfréquence du pôle GIP-CNFM de Lille (PLFM), ces projets ont permis de se confronter aux différents métiers de l'ingénieur hyperfréquence, i.e. conception, fabrication, test et validation, et intégration système. 


\section{Remerciements}

Ce travail a reçu le soutien du GIP-CNFM, du Ministère de l'Enseignement Supérieur de la Recherche et de l'Innovation et du projet IDEFI-FINMINA (16). Ce travail a été effectué au sein de la plate-forme hyperfréquence du pôle GIP-CNFM de Lille. Les auteurs remercient Lamine Kone pour les mesures effectuées en chambre anéchoïque.

\section{Références}

1. K. Wu, "MHz-Through-THz (MTT) Challenges and Opportunities [President's Column]," in IEEE Microwave Magazine, vol. 17, no. 2, pp. 8-10 (2016).

2. T. K. Sarkar, R. Mailloux, A. A. Oliner, M. Salazar-Palma and D. L. Sengupta, "The Evolution of Electromagnetic Waveguides: From Hollow Metallic Guides to Microwave Integrated Circuits," in History of Wireless, 1, Wiley-IEEE Press, pp. 543-566 (2016).

3. K. C. Gupta, T. Itoh, and A. A. Oliner, "Microwave and RF education-past, present, and future," IEEE Trans. Microw. Theory Tech., vol. 50, no. 3, pp. 1006-1014 (2002).

4. T. M. Weller, P. G. Flikkema, L. P. Dunleavy, H. C. Gordon, and R. E. Henning, "Educating tomorrow's RF/microwave engineer: A new undergraduate laboratory uniting circuit and system concepts," in Proc. Microw. Symp. Dig., 1998 IEEE MTT-S Int., vol. 2, pp. 563-566 (1998).

5. GIP-CNFM: Groupement d'Intérêt Public - Coordination Nationale pour la formation en Microélectronique et en nanotechnologies. Website: http://wwww.cnfm.fr.

6. Marketsandmarkets.com, Publishing Date: January 2017, Report Code: AS 2320

7. C. Li, V. M. Lubecke, O. Boric-Lubecke and J. Lin, "A Review on Recent Advances in Doppler Radar Sensors for Noncontact Healthcare Monitoring," IEEE Trans. Microw. Theory Tech., vol. 61, no. 5, pp. 2046-2060 (2013).

8. K. Haddadi, Mesure hyperfréquence des propriétés électromagnétiques de matériaux : $300 \mathrm{MHz}$ à $300 \mathrm{GHz}$, in Techniques de l'Ingénieur, TI Sciences et Techniques, dossier R1117, 31 pages, Paris, France (2016).

9. K. Haddadi and C. Loyez, "Millimeter-wave six-port IQ demodulator in $65 \mathrm{~nm}$ SOI CMOS technology," 2016 IEEE International Instrumentation and Measurement Technology Conference Proceedings, Taipei, pp. 1-5 (2016)

10. K. Haddadi and C. Loyez, "65 nm SOI CMOS $60 \mathrm{GHz}$ passive mixer for six-port technology," 2016 IEEE Topical Conference on Wireless Sensors and Sensor Networks (WiSNet), Austin, TX, pp. 5255 (2016).

11. S. Lallemand, K. Haddadi and C. Loyez, "A review of the six-port technique for metrology applications," 2018 22nd International Microwave and Radar Conference (MIKON), Poznan, pp. 7779 (2018).

12. A. E. Fellahi, K. Haddadi, D. Glay and T. Lasri, "Multiport Reflectometer Based on Subtractive Mixing," in IEEE Sensors Journal, vol. 15, no. 9, pp. 4729-4730 (2015).

13. K. Haddadi and T. Lasri, "Six-port technology for millimeter-wave radar and imaging applications," 2014 IEEE Topical Conference on Wireless Sensors and Sensor Networks (WiSNet), Newport Beach, CA, pp. 1-3 (2014).

14. K. Haddadi and T. Lasri, "Formulation for Complete and Accurate Calibration of Six-Port Reflectometer," in IEEE Transactions on Microwave Theory and Techniques, vol. 60, no. 3, pp. 574581 (2012).

15. K. Haddadi, M. Wang, D. Glay and T. Lasri, "Ultra wide-band four-port reflectometer using only two quadratic detectors," 2008 IEEE MTT-S International Microwave Symposium Digest, Atlanta, GA, USA, 2008, pp. 379-382 (2008).

16. IDEFI-FINMINA : Initiative d'Excellence - Formation Innovante en MIcroélectronique et Nanotechnologies, ANR-11-IDFI-0017. Website: http://www.cnfm.fr/VersionFrancaise/actualites/ FINMINA.htm 